\title{
DOA Estimation for Multiple Targets in MIMO Radar with Nonorthogonal Signals
}

\author{
Zhenxin Cao, ${ }^{1}$ Peng Chen $\left(\mathbb{D},{ }^{1}\right.$ Zhimin Chen, ${ }^{2}$ and Yi Jin ${ }^{3}$ \\ ${ }^{1}$ State Key Laboratory of Millimeter Waves, Southeast University, Nanjing 210096, China \\ ${ }^{2}$ School of Electronic and Information Engineering, Shanghai Dianji University, Shanghai 201306, China \\ ${ }^{3}$ Xian Branch of China Academy of Space Technology, Xi'an 710100, China \\ Correspondence should be addressed to Peng Chen; chenpengdsp@seu.edu.cn
}

Received 27 November 2017; Revised 26 June 2018; Accepted 8 July 2018; Published 15 July 2018

Academic Editor: Raffaele Solimene

Copyright (C) 2018 Zhenxin Cao et al. This is an open access article distributed under the Creative Commons Attribution License, which permits unrestricted use, distribution, and reproduction in any medium, provided the original work is properly cited.

\begin{abstract}
This paper addresses the direction of arrival (DOA) estimation problem in the colocated multiple-input multiple-output (MIMO) radar with nonorthogonal signals. The maximum number of targets that can be estimated is theoretically derived as $\operatorname{rank}\left\{\boldsymbol{R}_{s}\right\} N$, where $N$ denotes the number of receiving antennas and $\boldsymbol{R}_{s}$ is the cross-correlation matrix of the transmitted signals. Therefore, with the rank-deficient cross-correlation matrix, the maximum number that can be estimated is less than the radar with orthogonal signals. Then, a multiple signal classification- (MUSIC-) based algorithm is given for the nonorthogonal signals. Furthermore, the DOA estimation performance is also theoretically analyzed by the Carmér-Rao lower bound. Simulation results show that the nonorthogonality degrades the DOA estimation performance only in the scenario with the rank-deficient cross-correlation matrix.
\end{abstract}

\section{Introduction}

In the multiple-input multiple-output (MIMO) radar system [1-3], the waveform diversity can be used to improve the target detection and estimation performance [4-8]. In the existing works, many methods have been proposed to estimate the target direction of arrival (DOA). For example, in the colocated MIMO radar system, a reduced-dimension transformation is used to reduce the complexity in the DOA estimation based on the estimation of signal parameters via rotational invariance technique (ESPRIT) [9]; a computationally efficient DOA estimation algorithm is given for the monostatic MIMO radar based on the covariance matrix reconstruction in [10]; a joint DOA and direction of departure (DOD) estimation method based on ESPRIT is proposed in [11]. Additionally, in the coprime MIMO radar system, a reduced-dimension multiple signal classification (MUSIC) algorithm is proposed [12] for both DOA and DOD estimation; a combined unitary ESPRIT-based algorithm is given for the DOA estimation [13]. In the bistatic MIMO radar system, a joint DOD and DOA estimation method is also proposed in the scenario with an unknown spatially correlated noise [14].

In the existing papers about the MIMO radar systems, most papers are about the orthogonal waveforms to estimate the DOA, such as the waveforms adopted in [15-20]. However, it is difficult to generate the orthogonal signals in the practical radar systems, and the number of orthogonal waveforms is much less than that of nonorthogonal waveforms. Therefore, the traditional DOA estimation methods and results with the orthogonal signals must also be modified. In [21], the nonorthogonal waveforms are first proposed, and the method with prewhitening processing is proposed in [22]. However, to the best of our knowledge, the theoretical analysis of the nonorthogonal waveforms in the MIMO radar systems has not yet been addressed in the papers.

In this paper, the DOA estimation problem for multiple targets is addressed, and the system model of MIMO radar with nonorthogonal signals is given. Then, the maximum number of targets that can be estimated is derived according to the cross-correlation matrix of transmitted signals, and a MUSIC algorithm for the nonorthogonal signals is 
also proposed. Furthermore, the Carmér-Rao lower bound (CRLB) of DOA estimation with the nonorthogonal signals is also theoretically derived and shows that the estimation performance with nonorthogonal signals is only degraded in the scenario with rank-deficient cross-correlation matrix. To summarize, we make the following contributions:

(i) The MIMO radar system model with nonorthogonal waveforms: with considering the nonorthogonal waveforms, the system model of MIMO radar with DOA estimation is given

(ii) The theoretical maximum number of targets: with the subspace-based DOA estimation methods, the maximum number of targets that can be estimated is theoretically derived

(iii) The MUSIC-based method and CRLB for DOA estimation: to show the DOA estimation performance with nonorthogonal waveforms, the MUSICbased DOA estimation method is proposed, and the corresponding CRLB is theoretically derived.

Notations. $\mathbf{1}_{N}$ stands for a $N \times 1$ vector with all entries being $1 . \boldsymbol{I}_{N}$ denotes an $N \times N$ identity matrix. $\mathscr{E}\{\cdot\}$ denotes the expectation operation. $\mathscr{C} \mathcal{N}(\boldsymbol{a}, \boldsymbol{B})$ denotes the complex Gaussian distribution with the mean being $\boldsymbol{a}$ and the variance matrix being $\boldsymbol{B}$. $\|\cdot\|_{2}, \otimes, \odot, \operatorname{Tr}\{\cdot\}, \operatorname{vec}\{\cdot\},(\cdot)^{T}$, and $(\cdot)^{H}$ denote the $\ell_{2}$ norm, the Kronecker product, the Hadamard product, the trace of a matrix, the vectorization of a matrix, the matrix transpose, and the Hermitian transpose, respectively.

\section{System Model}

In this paper, the colocated MIMO radar $[8,23]$ is adopted, where the antenna numbers of transmitter and receiver are $M$ and $N$, respectively. We consider the DOA estimation problem for $K$ far-field targets. For the $M$ transmitting antennas, $s_{m}(t)$ denotes the waveform in the $m$ th antenna $(m=0,1, \ldots, M-1)$. In the traditional MIMO radar system, the waveforms $s_{m}(t)$ are orthogonal and $\int_{t} s_{m}(t) s_{m^{\prime}}(t) d t=$ $0\left(m \neq m^{\prime}\right)$. However, in this paper, the nonorthogonal waveforms are adopted, so we have $\int_{t} s_{m}(t) s_{m^{\prime}}(t) d t \neq 0(m \neq$ $\left.m^{\prime}\right)$. Additionally, $P$ pulses are transmitted in the MIMO radar, and the waveforms between pulses are the same. Then, during the $p$ th pulse $(p=0,1, \ldots, P-1)$, the signal in the $n$th receiving antenna $(n=0,1, \ldots, N-1)$ can be expressed as

$$
\begin{aligned}
r_{n}(p, t)= & \sum_{m=0}^{M-1} \sum_{k=0}^{K-1} \alpha_{k}(p) s_{m}(t) e^{-j(2 \pi / \lambda)\left(m d_{T}+n d_{R}\right) \cos \theta_{k}} \\
& +w_{n}(p, t)
\end{aligned}
$$

where

$$
\boldsymbol{\theta} \triangleq\left(\theta_{0}, \ldots, \theta_{K-1}\right)^{T},
$$

where $\theta_{k}$ denotes the DOA of the $k$ th target $(k=0,1, \ldots, K-$ $1)$ and $w_{n}(p, t)$ denotes the additive white Gaussian noise
(AWGN) with the variance being $\sigma_{n}^{2} \cdot \lambda$ denotes the wavelength of the transmitted waveform. $d_{T}$ and $d_{R}$ denote the antenna spacing in transmitter and receiver, respectively. $\alpha_{k}(p)$ denotes the fading coefficient of the $k$ th target's radar cross section (RCS) during the pth pulse. We assume that $\alpha_{k}(p)$ is a type of Swerling II RCS and follows the independent and identical distribution between pulses. After the matched filtering processing for the $m$ th signal during the $p$ th pulse, we can obtain the sampled signal as

$$
\begin{aligned}
& r_{n, p, m} \triangleq \int_{t} r_{n}(p, t) s_{m}^{H}(t) d t=\sum_{m^{\prime}=0}^{M-1} \sum_{k=0}^{K-1} \alpha_{k}(p) \\
& \cdot e^{-j(2 \pi / \lambda)\left(m^{\prime} d_{T}+n d_{R}\right) \cos \theta_{k}} \int_{t} s_{m^{\prime}}(t) s_{m}^{H}(t) d t \\
& +\int_{t} w_{n}(p, t) s_{m}^{H}(t) d t .
\end{aligned}
$$

After the simplifications, $r_{n, p, m}$ can be rewritten as

$$
r_{n, p, m}=b_{n}^{T}(\boldsymbol{\theta}) \operatorname{diag}\{\boldsymbol{\alpha}(p)\} \boldsymbol{A}^{T} \boldsymbol{R}_{s}[m]+w_{n, m}(p),
$$

where $\boldsymbol{R}_{s}$ denotes the cross-correlation matrix of the transmitted signals. The $m$ th row and $m^{\prime}$ th column entry of $\boldsymbol{R}_{s}$ is defined as

$$
\boldsymbol{R}_{s, m, m^{\prime}} \triangleq \int_{t} s_{m}(t) s_{m^{\prime}}^{H}(t) d t,
$$

and $\boldsymbol{R}_{s}[m]$ denotes the $m$ th column of $\boldsymbol{R}_{s} \cdot \operatorname{diag}\{\boldsymbol{\alpha}(p)\}$ denotes a diagonal matrix with all the entries from the vector $\boldsymbol{\alpha}(p)$, and the steering vector in the transmitter is defined as

$$
\boldsymbol{a}\left(\theta_{k}\right) \triangleq\left[e^{-j(2 \pi / \lambda) 0 d_{T} \cos \theta_{k}}, \ldots, e^{-j(2 \pi / \lambda)(M-1) d_{T} \cos \theta_{k}}\right]^{T} .
$$

The other symbols are defined as follows:

$$
\begin{aligned}
\boldsymbol{A} & \triangleq\left[\boldsymbol{a}\left(\theta_{0}\right), \boldsymbol{a}\left(\theta_{1}\right), \ldots, \boldsymbol{a}\left(\theta_{K-1}\right)\right], \\
w_{n, m}(p) & \triangleq \int_{t} w_{n}(p, t) s_{m}^{H}(t) d t, \\
\boldsymbol{\alpha}(p) & \triangleq\left[\alpha_{0}(p), \ldots, \alpha_{K-1}(p)\right]^{T}, \\
b_{n}(\boldsymbol{\theta}) & \triangleq\left[e^{-j(2 \pi / \lambda) n d_{R} \cos \theta_{0}}, \ldots, e^{-j(2 \pi / \lambda) n d_{R} \cos \theta_{K-1}}\right]^{T} .
\end{aligned}
$$

Since the nonorthogonal signals are transmitted in the MIMO radar, the off-diagonal entries of $\boldsymbol{R}_{s}$ are not all zeros.

We can collect all the received signals in (4) into a matrix $\mathbf{R}(p)$ as

$$
\boldsymbol{R}(p)=\boldsymbol{B} \operatorname{diag}\{\boldsymbol{\alpha}(p)\} \boldsymbol{A}^{T} \boldsymbol{R}_{\boldsymbol{s}}+\boldsymbol{W}(p),
$$

where the $n$th row and $m$ th column of $\boldsymbol{R}(p)$ is $r_{n, p, m}$ and the $n$th row and $m$ th column of $\boldsymbol{W}(p)$ is $w_{n, m}(p)$.

$$
\boldsymbol{B} \triangleq\left[b_{0}(\boldsymbol{\theta}), \ldots, b_{N-1}(\boldsymbol{\theta})\right]^{T}=\left[\boldsymbol{b}\left(\theta_{0}\right), \ldots, \boldsymbol{b}\left(\theta_{K-1}\right)\right],
$$

where the steering vector in the receiver is defined as

$$
\boldsymbol{b}\left(\theta_{k}\right) \triangleq\left[e^{-j(2 \pi / \lambda) 0 d_{R} \cos \theta_{k}}, \ldots, e^{-j(2 \pi / \lambda)(N-1) d_{R} \cos \theta_{k}}\right]^{T} .
$$

In this paper, we consider the DOA estimation with orthogonal waveforms, and the system model is given in (8), where the unknown DOAs are collected by matrices $\mathbf{A}$ and $\mathbf{B}$. 


\section{DOA Estimation}

With the received signal $\boldsymbol{R}(p)$, the DOA can be estimated, and the estimation performance with the nonorthogonal signals is analyzed in this section. The vectorization form of (8) can be expressed as

$$
\boldsymbol{r}(p) \triangleq \operatorname{vec}\{\boldsymbol{R}(p)\}=\boldsymbol{T} \boldsymbol{\alpha}(p)+\boldsymbol{w}(p)
$$

where

$$
\begin{aligned}
\boldsymbol{T} \triangleq & \left(\boldsymbol{R}_{s}^{T} \boldsymbol{A} \otimes \mathbf{1}_{N}\right) \odot\left(\mathbf{1}_{M} \otimes \boldsymbol{B}\right) \\
= & {\left[\boldsymbol{R}_{s}^{T} \boldsymbol{a}\left(\theta_{0}\right) \otimes \mathbf{1}_{N}, \ldots, \boldsymbol{R}_{s}^{T} \boldsymbol{a}\left(\theta_{K-1}\right) \otimes \mathbf{1}_{N}\right] } \\
& \odot\left[\mathbf{1}_{M} \otimes \boldsymbol{b}\left(\theta_{0}\right), \ldots, \mathbf{1}_{M} \otimes \boldsymbol{b}\left(\theta_{K-1}\right)\right] \\
= & {\left[\boldsymbol{t}_{0}, \ldots, \boldsymbol{t}_{K-1}\right], }
\end{aligned}
$$

and $\otimes$ denotes the Kronecker product, $\odot$ denotes the Hadamard product (entry-wise product),

$$
\boldsymbol{w}(p) \triangleq \operatorname{vec}\{\boldsymbol{W}(p)\}
$$

and

$$
\boldsymbol{t}_{k} \triangleq \boldsymbol{R}_{s}^{T} \boldsymbol{a}\left(\theta_{k}\right) \otimes \boldsymbol{b}\left(\theta_{k}\right)
$$

denotes the $k$ th column of $\boldsymbol{T}$. Moreover, the noise $\boldsymbol{w}(p)$ follows the zero-mean Gaussian distribution with the covariance matrix being

$$
\mathscr{E}\left\{\boldsymbol{w}(p) \boldsymbol{w}^{H}(p)\right\}=\sigma_{n}^{2}\left(\boldsymbol{R}_{s}^{T} \otimes \boldsymbol{I}_{N}\right) .
$$

In the following proposition, we will theoretically derive the maximum number of targets that can be estimated by the nonorthogonal waveforms in the MIMO radar system.

Proposition 1. In the colocated MIMO radar system with $M$ transmitting antennas and $N$ receiving antennas, the maximum number of targets in the DOA estimation using the subspace-based methods is $\operatorname{rank}\left\{\boldsymbol{R}_{s}\right\} N$, where $\boldsymbol{R}_{s}$ denotes the cross-correlation matrix of transmitted signals. Since $\operatorname{rank}\left\{\boldsymbol{R}_{s}\right\} \leq M$, the maximum number $\operatorname{rank}\left\{\boldsymbol{R}_{s}\right\} N \leq M N$.

Proof. (1) When $\boldsymbol{R}_{s}$ is full $\operatorname{rank} \operatorname{rank}\left\{\boldsymbol{R}_{s}\right\}=M$, we have

$$
\operatorname{rank}\left\{\sigma_{n}^{2}\left(\boldsymbol{R}_{s}^{T} \otimes \boldsymbol{I}_{N}\right)\right\}=M N
$$

Therefore, the matrix $\left(\boldsymbol{R}_{s}^{T} \otimes \boldsymbol{I}_{N}\right)$ is invertible, and the AWGN can be obtained by the following prewhitening processing:

$$
\boldsymbol{y}(p) \triangleq \mathbf{Q} \boldsymbol{r}(p)=\boldsymbol{s}_{T}+\boldsymbol{z}(p)
$$

where

$$
\mathbf{Q} \triangleq\left(\boldsymbol{R}_{s}^{T} \otimes \boldsymbol{I}_{N}\right)^{-1 / 2}
$$

denotes the prewhitening matrix, and

$$
\boldsymbol{s}_{T} \triangleq \mathbf{Q T} \boldsymbol{\alpha}(p)
$$

$z(p)$ denotes the AWGN with the variance matrix being $\sigma_{n}^{2} \boldsymbol{I}_{M N}$. Then, the cross-correlation matrix of $\boldsymbol{s}_{T}$ can be obtained as

$$
\boldsymbol{R}_{T} \triangleq \mathscr{E}\left\{\boldsymbol{s}_{T} \boldsymbol{s}_{T}^{H}\right\}
$$

and the dimension of signal subspace can be calculated as

$$
\begin{aligned}
D_{T} & \triangleq \operatorname{rank}\left\{\boldsymbol{R}_{T}\right\} \\
& \leq \min \left\{M N, \operatorname{rank}\left\{\boldsymbol{R}_{s}^{T} \boldsymbol{A}\right\} \operatorname{rank}\{\boldsymbol{B}\}, K\right\} .
\end{aligned}
$$

With $K \leq M N$, we have

$$
D_{T} \leq \min \{\min \{M, K\} \min \{N, K\}, K\}=K \leq M N .
$$

Therefore, when the subspace-based methods, such as MUSIC algorithm, are adopted to estimate the DOA, the maximum number of targets that can be estimated is $K \leq$ $M N$.

(2) When $\boldsymbol{R}_{s}$ is not full $\operatorname{rank}\left\{\boldsymbol{R}_{s}\right\}<M$, the prewhitening matrix in (17) is irreversible and cannot be adopted, so we must find other prewhitening matrices. Since the covariance matrix of noise is a positive-semidefinite normal matrix, we have the following decomposition:

$$
\boldsymbol{R}_{s}^{T} \otimes \boldsymbol{I}_{N}=\boldsymbol{U} \boldsymbol{D} \boldsymbol{U}^{H}
$$

where $\boldsymbol{U}$ is a unitary matrix composed by the eigenvectors of $\boldsymbol{R}_{s}^{T} \otimes \boldsymbol{I}_{N}$ and $\boldsymbol{D}$ is a nonnegative real diagonal matrix with the diagonal entries being the eigenvalues of $\boldsymbol{R}_{s}^{T} \otimes \boldsymbol{I}_{N}$. By collecting the nonzero entries of $\boldsymbol{D}$ into a vector $\boldsymbol{d} \in \mathbb{C}^{z \times 1}$ and the corresponding eigenvectors into a matrix $\boldsymbol{U}_{d}$, where

$$
z \triangleq \operatorname{rank}\left\{\boldsymbol{R}_{s}^{T} \otimes \boldsymbol{I}_{N}\right\}=N \operatorname{rank}\left\{\boldsymbol{R}_{s}\right\},
$$

we can obtain the following prewhitening matrix:

$$
\boldsymbol{Q}^{\prime} \triangleq\left[\operatorname{diag}^{-1 / 2}\{\boldsymbol{d}\}, \mathbf{0}_{z \times(M N-z)}\right]\left[\boldsymbol{U}_{d}, \boldsymbol{U}_{0}\right]^{H},
$$

where $\boldsymbol{U}_{0}$ are the matrices of eigenvectors corresponding to the zero eigenvalues. Then, we have the following prewhitening processing:

$$
\boldsymbol{y}^{\prime}(p)=\mathbf{Q}^{\prime} \boldsymbol{r}(p)=\mathbf{Q}^{\prime} \boldsymbol{T} \boldsymbol{\alpha}(p)+\boldsymbol{z}^{\prime}(p),
$$

where the noise is

$$
\boldsymbol{z}^{\prime}(p) \triangleq \mathbf{Q}^{\prime} \boldsymbol{w}(p)
$$

and the covariance matrix of noise is

$$
\mathscr{E}\left\{z^{\prime}(p) z^{\prime H}(p)\right\}=\sigma_{n}^{2} \boldsymbol{I}_{z} .
$$

Then, the cross-correlation matrix of the echoed signals can be obtained as

$$
\boldsymbol{R}_{T}^{\prime} \triangleq\left[\boldsymbol{Q}^{\prime} \boldsymbol{T} \boldsymbol{\alpha}(p)\right]\left[\boldsymbol{Q}^{\prime} \boldsymbol{T} \boldsymbol{\alpha}(p)\right]^{H},
$$

and the corresponding rank is

$$
D_{T}^{\prime} \triangleq \operatorname{rank}\left\{\boldsymbol{R}_{T}^{\prime}\right\} \leq \min \{z, K\} .
$$


With $\operatorname{rank}\left\{\boldsymbol{R}_{s}\right\}<M$, the maximum number of targets that can be estimated is $\operatorname{rank}\left\{\boldsymbol{R}_{s}\right\} N$, so when the nonorthogonal signals reduce the rank of the cross-correlation matrix $\boldsymbol{R}_{s}$, the maximum number of targets that can be estimated also decreases.

With Proposition 1, we find that the maximum number of targets that can be estimated is $\operatorname{rank}\left\{\boldsymbol{R}_{s}\right\} N$, so, with $K<$ $\operatorname{rank}\left\{\boldsymbol{R}_{s}\right\} N$, we propose a MUSIC-based method to estimate the DOAs. After the prewhitening processes, the MUSIC spatial spectrum can be obtained as

$$
s(\theta)=\frac{1}{c^{H}(\theta) S_{N} S_{N}^{H} c(\theta)},
$$

where

$$
\boldsymbol{c}(\theta) \triangleq \boldsymbol{Q}^{\prime}\left[\boldsymbol{R}_{s}^{T} \boldsymbol{a}(\theta) \otimes \boldsymbol{b}(\theta)\right],
$$

and $S_{N}$ is the matrix of noise subspace formulated by the $(M N-K)$ eigenvectors corresponding to the minimum eigenvalues of the received signals $y^{\prime}(p)$ after the prewhitening. The DOAs can be estimated as

$$
\widehat{\boldsymbol{\theta}}=\left(\widehat{\theta}_{0}, \widehat{\theta}_{1}, \ldots, \widehat{\theta}_{K-1}\right)^{T}
$$

by selecting the peak positions of the MUSIC spatial spectrum.

\section{Carmér-Rao Lower Bound}

A vector $\boldsymbol{r}$ can be formulated by collecting the signals from all receiving antennas and pulses.

$$
r=\left\{I_{P} \otimes T\right\} \alpha+w,
$$

where

$$
\begin{aligned}
\boldsymbol{r} & \triangleq\left[\boldsymbol{r}^{T}(0), \boldsymbol{r}^{T}(1), \ldots, \boldsymbol{r}^{T}(P-1)\right]^{T}, \\
\boldsymbol{\alpha} & \triangleq\left[\boldsymbol{\alpha}^{T}(0), \boldsymbol{\alpha}^{T}(1), \ldots, \boldsymbol{\alpha}^{T}(P-1)\right]^{T}, \\
\boldsymbol{w} & \triangleq\left[\boldsymbol{w}^{T}(0), \boldsymbol{w}^{T}(1), \ldots, \boldsymbol{w}^{T}(P-1)\right]^{T} .
\end{aligned}
$$

By assuming that the fading coefficients follow the zero-mean Gaussian distribution

$$
\boldsymbol{\alpha} \sim \mathscr{C} \cdot \mathcal{N}\left(\mathbf{0}_{P K}, \alpha^{2} \boldsymbol{I}_{P K}\right),
$$

$\boldsymbol{r}$ also follows the zero-mean Gaussian distribution:

$$
\boldsymbol{r} \sim f(\boldsymbol{r} ; \boldsymbol{\theta})=\frac{1}{\pi^{M N P} \operatorname{det}\left(\boldsymbol{C}_{r}\right)} e^{-\boldsymbol{r}^{H} \boldsymbol{C}_{r}^{-1} \boldsymbol{r}}
$$

with the covariance matrix being

$$
\boldsymbol{C}_{r} \triangleq \sigma_{n}^{2} \boldsymbol{I}_{P} \otimes\left(\boldsymbol{R}_{s}^{T} \otimes \boldsymbol{I}_{N}\right)+\alpha^{2} \boldsymbol{I}_{P} \otimes \boldsymbol{T} \boldsymbol{T}^{H} .
$$

With the receiving signal $\boldsymbol{r}$, the DOA can be estimated by the MUSIC algorithm proposed in this paper, and the estimation performance is bounded by the CRLB.

$$
\mathscr{E}\left\{\|\boldsymbol{\theta}-\widehat{\boldsymbol{\theta}}\|_{2}^{2}\right\} \geq g(\boldsymbol{\theta}) \triangleq \operatorname{Tr}\left\{\boldsymbol{F}^{-1}\right\},
$$

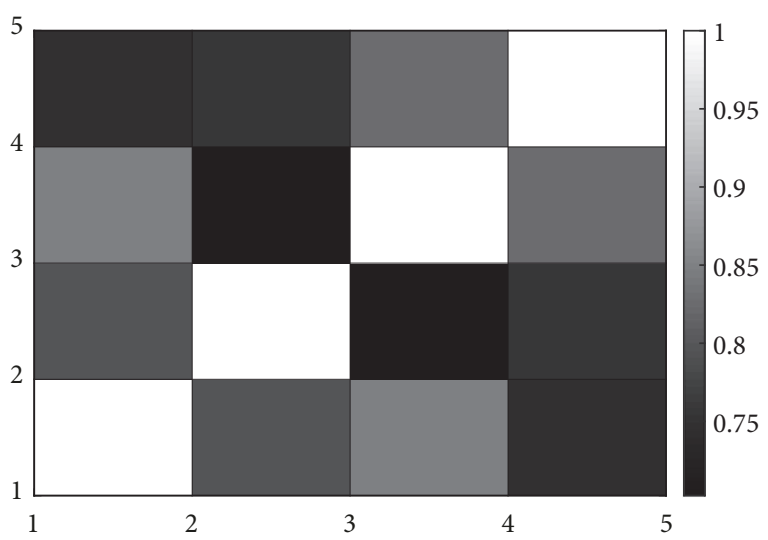

FIgURE 1: The illustration of $\boldsymbol{R}_{s}$.

where $g(\boldsymbol{\theta})$ denotes the CRLB of DOA estimation and $\boldsymbol{F}$ denotes the Fisher information matrix (FIM). The entry in the $i$ th row and $j$ th column $(i=0,1, \ldots, K-1, j=0,1, \ldots, K-1)$ of the FIM can be obtained as

$$
F_{i, j}=-\mathscr{E}\left\{\frac{\partial^{2} \ln f(\boldsymbol{r} ; \boldsymbol{\theta})}{\partial \theta_{i} \partial \theta_{j}}\right\},
$$

and the detailed expressions of FIM are given in the Appendix. Similarity, with the orthogonal waveforms, we have $\boldsymbol{R}_{s}=\boldsymbol{I}_{M}$, and the corresponding CRLB can be also obtained.

\section{Simulation Results}

In this section, the simulation results are given, and the parameters are set as follows: the numbers of transmitting antennas, receiving antennas, and pulses are $M=5, N=10$, and $P=10$, respectively; the carrier frequency is $10 \mathrm{GHz}$; the wavelength is $\lambda=0.03 \mathrm{~m}$; the antenna spacing is $d_{T}=d_{R}=$ $\lambda / 2$. In Figure 2, the MUSIC algorithm is used to estimate the DOA of 3 targets, where the DOAs are 0.1881, 1.0461, and 1.4071, respectively. With the signal-to-noise ratio (SNR) being $25 \mathrm{~dB}$, the MUSIC algorithm is used to estimate the DOA, and $\boldsymbol{R}_{s}$ is shown in Figure 1. After $10^{5}$ Monte Carlo experiments, the root-mean-square error (RMSE) of DOA estimation can be obtained as 0.0103 . The rank of waveforms adopted in Figure 2 is $M$, but the rank of waveforms adopted in Figure 3 is 1 . Therefore, the estimation performance is degraded in the scenario with rank-deficient waveforms.

In the scenario with nonorthogonal signals, Figure 4 shows the DOA estimation performance using the MUSIC method with different types of transmitted signals, including the orthogonal signals, the full-rank nonorthogonal signals ( $\left.\operatorname{rank}\left\{\boldsymbol{R}_{s}\right\}=5\right)$, and the rank-deficient nonorthogonal signals $\left(\operatorname{rank}\left\{\boldsymbol{R}_{s}\right\}=1\right)$. As shown in this figure, with increasing the SNR of received signals, the DOA estimation performance is improved and approaches the CRLBs with both orthogonal and nonorthogonal signals. However, the nonorthogonal signals achieve worse estimation performance compared with 


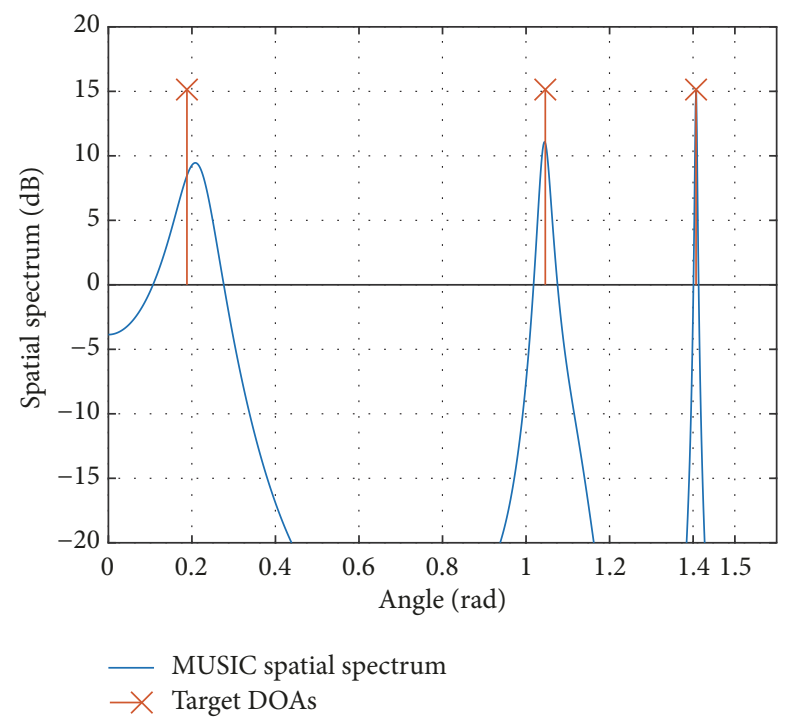

Figure 2: The MUSIC algorithm to estimate DOA (full rank).

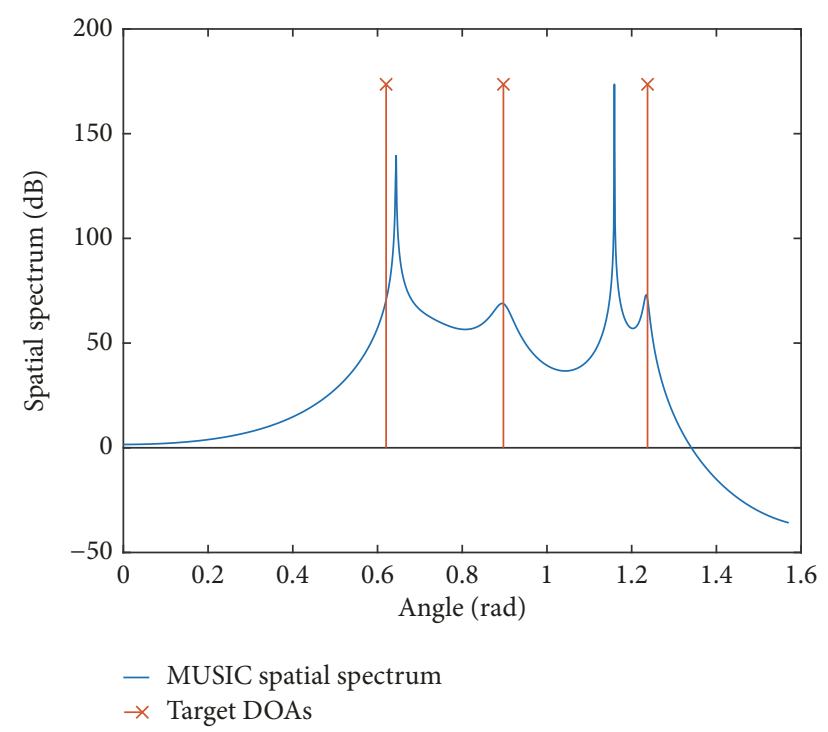

FIgURE 3: The MUSIC algorithm to estimate DOA ( $\mathrm{rank}=1)$.

the orthogonal signals, especially in the scenario with rankdeficient waveforms. Moreover, we also show the DOA estimation performance using the state-of-art direction finding method. In [24], by discretizing the detection area into grids, the simultaneous orthogonal matching pursuit (SOMP) is proposed, and the DOA estimation can be formulated as a sparse reconstruction problem. Figure 5 shows the DOA estimation with different waveforms, and the better estimation performance can be achieved by both the full-rank and the orthogonal waveforms compared with the rank-deficient waveform. Therefore, the nonorthogonality degrades the DOA estimation performance only in the scenario with rankdeficient waveforms.

In Figure 6, we show the DOA estimation performance with different numbers of targets and the MUSIC method

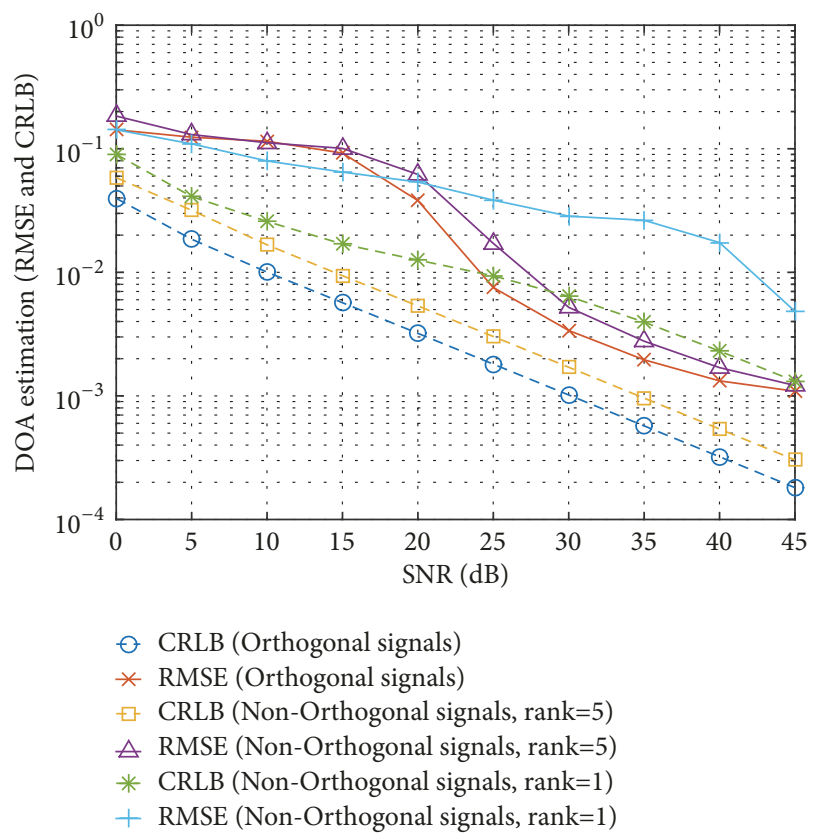

FIGURE 4: DOA estimation performance with different values of SNR (MUSIC).

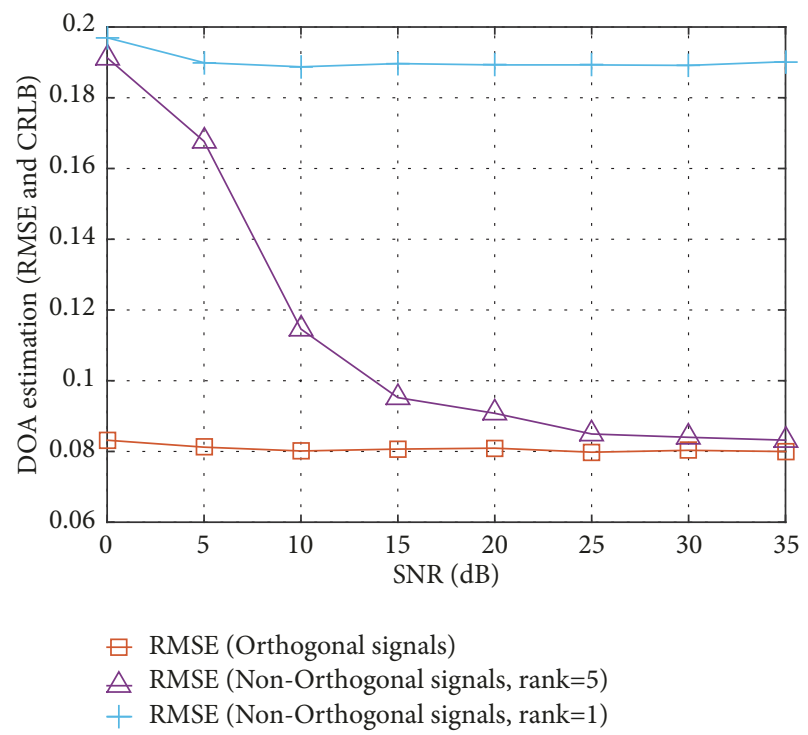

FIGURE 5: DOA estimation performance with different values of SNR (SOMP).

is adopted, where SNR is $30 \mathrm{~dB}$. As shown in this figure, the estimation performance is degraded by more targets, and full-rank cross-correlation matrix can achieve better estimation performance than the rank-deficient one. The estimation performance can achieve the CRLB in the scenario with fewer targets. Therefore, in the DOA estimation scenario with multiple targets, the nonorthogonality gives worse estimation performance, especially with the rankdeficient nonorthogonal signals. In the future work about waveform design of MIMO radar systems, when the full-rank waveforms are adopted, the DOA estimation performance 


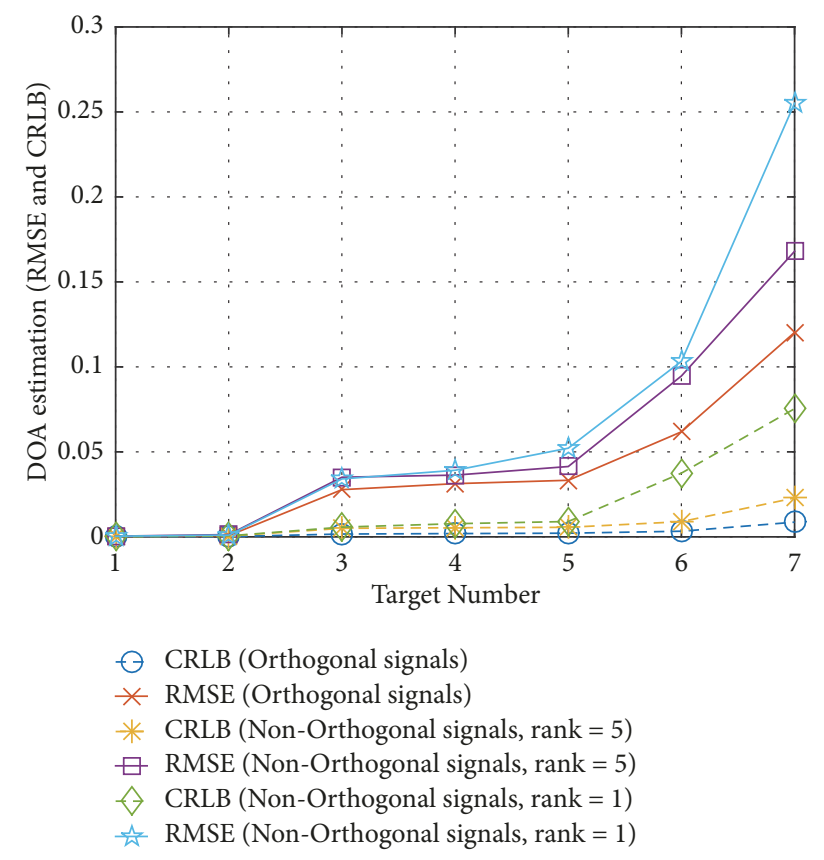

FIGURE 6: DOA estimation performance with different numbers of targets.

cannot be degraded greatly, so we can choose the full-rank nonorthogonal waveforms with more freedom.

\section{Conclusion}

In this paper, the DOA estimation problem with nonorthogonal signals has been addressed in the MIMO radar system. The maximum number of targets, which is limited by the rank of the cross-correlation matrix, has been analyzed and given. Then, the MUSIC algorithm for nonorthogonal signals has also been given, and the CRLB for nonorthogonal signals has been derived. Simulation results show that the estimation performance is mainly degraded by the rankdeficient cross-correlation matrix of the transmitted signals. Future work will focus on the waveform design using the fullrank nonorthogonal waveforms in the MIMO radar systems.

\section{Appendix}

\section{The Detailed Expressions of FIM}

The entry in the $i$ th row and $j$ th column of the FIM can be expressed as

$$
\begin{aligned}
F_{i, j} & =-\mathscr{E}\left\{\frac{\partial^{2} \ln f(\boldsymbol{r} ; \boldsymbol{\theta})}{\partial \theta_{i} \partial \theta_{j}}\right\} \\
& =\operatorname{Tr}\left\{\boldsymbol{C}_{r}^{-1} \frac{\partial \boldsymbol{C}_{r}}{\partial \theta_{j}} \boldsymbol{C}_{r}^{-1} \frac{\partial \boldsymbol{C}_{r}}{\partial \theta_{i}}\right\}+\operatorname{Tr}\left\{\boldsymbol{C}_{r}^{-1} \frac{\partial^{2} \boldsymbol{C}_{r}}{\partial \theta_{i} \partial \theta_{j}}\right\},
\end{aligned}
$$

where $\partial \boldsymbol{C}_{r} / \partial \theta_{i}$ can be simplified as

$$
\frac{\partial \boldsymbol{C}_{r}}{\partial \theta_{i}}=\alpha^{2} \boldsymbol{I}_{P} \otimes \frac{\partial \boldsymbol{T} \boldsymbol{T}^{H}}{\partial \theta_{i}}
$$

$$
=\alpha^{2} \boldsymbol{I}_{P} \otimes\left(\frac{\partial \boldsymbol{T}}{\partial \theta_{i}} \boldsymbol{T}^{H}+\boldsymbol{T} \frac{\partial \boldsymbol{T}^{H}}{\partial \theta_{i}}\right)
$$

where

$$
\begin{aligned}
& \frac{\partial \boldsymbol{T}}{\partial \theta_{i}}=\left[\mathbf{0}_{M N \times(i-1)}, \frac{\partial \boldsymbol{t}_{i}}{\partial \theta_{i}}, \mathbf{0}_{M N \times(K-i)}\right], \\
& \frac{\partial \boldsymbol{t}_{i}}{\partial \theta_{i}}=\boldsymbol{R}_{s}^{T} \frac{\partial \boldsymbol{a}\left(\theta_{i}\right)}{\partial \theta_{i}} \otimes \boldsymbol{b}\left(\theta_{i}\right)+\boldsymbol{R}_{s}^{T} \boldsymbol{a}\left(\theta_{i}\right) \otimes \frac{\partial \boldsymbol{b}\left(\theta_{i}\right)}{\partial \theta_{i}} .
\end{aligned}
$$

Additionally, we can obtain

$$
\frac{\partial \boldsymbol{a}\left(\theta_{i}\right)}{\partial \theta_{i}}=j \frac{2 \pi}{\lambda} d_{T} \sin \theta_{i}\left(\boldsymbol{\eta}_{M} \odot \boldsymbol{a}\left(\theta_{i}\right)\right) .
$$

And

$$
\boldsymbol{\eta}_{M} \triangleq[0,1, \ldots, M-1]^{T}
$$

Using the same method, the derivative of $\boldsymbol{b}\left(\theta_{i}\right)$ can be also obtained as

$$
\frac{\partial \boldsymbol{b}\left(\theta_{i}\right)}{\partial \theta_{i}}=j \frac{2 \pi}{\lambda} d_{R} \sin \theta_{i}\left(\boldsymbol{\eta}_{N} \odot \boldsymbol{b}\left(\theta_{i}\right)\right) .
$$

The second-order derivative of $\boldsymbol{C}_{r}$ can be expressed as

$$
\begin{aligned}
& \frac{\partial^{2} \boldsymbol{C}_{r}}{\partial \theta_{i} \partial \theta_{j}} \quad \\
& =\alpha^{2} \boldsymbol{I}_{P} \otimes\left[\frac{\partial\left(\left(\partial \boldsymbol{T} / \partial \theta_{i}\right) \boldsymbol{T}^{H}\right)}{\partial \theta_{j}}+\frac{\partial\left(\boldsymbol{T}\left(\partial \boldsymbol{T}^{H} / \partial \theta_{i}\right)\right)}{\partial \theta_{j}}\right] \\
& =\alpha^{2} \boldsymbol{I}_{P} \\
& \qquad\left[\frac{\partial^{2} \boldsymbol{T}}{\partial \theta_{i} \partial \theta_{j}} \boldsymbol{T}^{H}+\frac{\partial \boldsymbol{T}}{\partial \theta_{i}} \frac{\partial \boldsymbol{T}^{H}}{\partial \theta_{j}}+\frac{\partial \boldsymbol{T}}{\partial \theta_{j}} \frac{\partial \boldsymbol{T}^{H}}{\partial \theta_{i}}+\boldsymbol{T} \frac{\partial^{2} \boldsymbol{T}^{H}}{\partial \theta_{i} \partial \theta_{j}}\right] \\
& = \begin{cases}\mathbf{0}_{M N P}, & i \neq j \\
\alpha^{2} \boldsymbol{I}_{P} \otimes\left(\frac{\partial^{2} \boldsymbol{t}_{i}}{\partial^{2} \theta_{i}} \boldsymbol{t}_{i}^{H}+2 \frac{\partial \boldsymbol{t}_{i}}{\partial \theta_{i}} \frac{\partial \boldsymbol{t}_{i}^{H}}{\partial \theta_{i}}+\boldsymbol{t}_{i} \frac{\partial^{2} \boldsymbol{t}_{i}^{H}}{\partial^{2} \theta_{i}}\right), & i=j\end{cases}
\end{aligned}
$$

where

$$
\begin{aligned}
\frac{\partial^{2} \boldsymbol{t}_{i}}{\partial^{2} \theta_{i}}= & \frac{\partial\left[\boldsymbol{R}_{s}^{T}\left(\partial \boldsymbol{a}\left(\theta_{i}\right) / \partial \theta_{i}\right) \otimes \boldsymbol{b}\left(\theta_{i}\right)\right]}{\partial \theta_{i}} \\
& +\frac{\partial\left[\boldsymbol{R}_{s}^{T} \boldsymbol{a}\left(\theta_{i}\right) \otimes\left(\partial \boldsymbol{b}\left(\theta_{i}\right) / \partial \theta_{i}\right)\right]}{\partial \theta_{i}} \\
= & \boldsymbol{R}_{s}^{T} \frac{\partial^{2} \boldsymbol{a}\left(\theta_{i}\right)}{\partial^{2} \theta_{i}} \otimes \boldsymbol{b}\left(\theta_{i}\right)+2 \boldsymbol{R}_{s}^{T} \frac{\partial \boldsymbol{a}\left(\theta_{i}\right)}{\partial \theta_{i}} \\
& \otimes \frac{\partial \boldsymbol{b}\left(\theta_{i}\right)}{\partial \theta_{i}}+\boldsymbol{R}_{s}^{T} \boldsymbol{a}\left(\theta_{i}\right) \otimes \frac{\partial^{2} \boldsymbol{b}\left(\theta_{i}\right)}{\partial^{2} \theta_{i}}
\end{aligned}
$$




$$
\begin{aligned}
\frac{\partial^{2} \boldsymbol{a}\left(\theta_{i}\right)}{\partial^{2} \theta_{i}}= & j \frac{2 \pi}{\lambda} d_{T} \frac{\partial \sin \theta_{i} \boldsymbol{\eta}_{M} \odot \boldsymbol{a}\left(\theta_{i}\right)}{\partial \theta_{i}} \\
= & j \frac{2 \pi}{\lambda} d_{T} \boldsymbol{\eta}_{M} \\
& \odot\left(\boldsymbol{a}\left(\theta_{i}\right) \cos \theta_{i}+\frac{\partial \boldsymbol{a}\left(\theta_{i}\right)}{\partial \theta_{i}} \sin \theta_{i}\right), \\
\frac{\partial^{2} \boldsymbol{b}\left(\theta_{i}\right)}{\partial^{2} \theta_{i}}= & j \frac{2 \pi}{\lambda} d_{R} \boldsymbol{\eta}_{N} \\
& \odot\left(\boldsymbol{a}\left(\theta_{i}\right) \cos \theta_{i}+\frac{\partial \boldsymbol{a}\left(\theta_{i}\right)}{\partial \theta_{i}} \sin \theta_{i}\right) .
\end{aligned}
$$

\section{Conflicts of Interest}

The authors declare that there are no conflicts of interest regarding the publication of this paper.

\section{Acknowledgments}

This work was supported in part by the National Natural Science Foundation of China (Grants nos. 61471117 and 61601281), the Open Program of the State Key Laboratory of Millimeter Waves (Southeast University) (Grant no. Z201804), and the Fundamental Research Funds for the Central Universities (Southeast University).

\section{References}

[1] P. Chen, C. Qi, and L. Wu, "Antenna placement optimisation for compressed sensing-based distributed MIMO radar," IET Radar, Sonar \& Navigation, vol. 11, no. 2, pp. 285-293, 2017.

[2] J. Li, P. Stoica, L. Xu, and W. Roberts, "On parameter identifiability of MIMO radar," IEEE Signal Processing Letters, vol. 14, no. 12, pp. 968-971, 2007.

[3] I. Bekkerman and J. Tabrikian, "Target detection and localization using MIMO radars and sonars," IEEE Transactions on Signal Processing, vol. 54, no. 10, pp. 3873-3883, 2006.

[4] J. Li and P. Stoica, "MIMO radar with colocated antennas," IEEE Signal Processing Magazine, vol. 24, no. 5, pp. 106-114, 2007.

[5] P. Chen and L. Wu, "System optimization for temporal correlated cognitive radar with EBPSK-based MCPC signal," Mathematical Problems in Engineering, vol. 2015, Article ID 302083, pp. 1-10, 2015.

[6] P. Chen, L. Wu, and C. Qi, "Waveform optimization for target scattering coefficients estimation under detection and peak-toaverage power ratio constraints in cognitive radar," Circuits, Systems and Signal Processing, vol. 35, no. 1, pp. 163-184, 2016.

[7] M. Haghnegahdar, S. Imani, S. A. Ghorashi, and E. Mehrshahi, "SINR Enhancement in Colocated MIMO Radar Using Transmit Covariance Matrix Optimization," IEEE Signal Processing Letters, vol. 24, no. 3, pp. 339-343, 2017.

[8] P. Chen, L. Zheng, X. Wang, H. Li, and L. Wu, "Moving target detection using colocated MIMO radar on multiple distributed moving platforms," IEEE Transactions on Signal Processing, vol. 65, no. 17, pp. 4670-4683, 2017.
[9] X. Zhang and D. Xu, "Low-complexity ESPRIT-based DOA estimation for colocated MIMO radar using reduced-dimension transformation," IEEE Electronics Letters, vol. 47, no. 4, pp. 283284, 2011.

[10] Y. Zhang, G. Zhang, and X. Wang, "Computationally efficient DOA estimation for monostatic MIMO radar based on covariance matrix reconstruction," IEEE Electronics Letters, vol. 53, no. 2, pp. 111-113, 2017.

[11] D. Oh, Y.-C. Li, J. Khodjaev, J.-W. Chong, and J.-H. Lee, "Joint estimation of direction of departure and direction of arrival for multiple-input multiple-output radar based on improved joint ESPRIT method," IET Radar, Sonar \& Navigation, vol. 9, no. 3, pp. 308-317, 2015.

[12] Y. Jia, X. Zhong, Y. Guo, and W. Huo, "DOA and DOD estimation based on bistatic MIMO radar with co-prime array," in Proceedings of the 2017 IEEE Radar Conference, RadarConf 2017, pp. 0394-0397, Seattle, Wash, USA, May 2017.

[13] J. Li, D. Jiang, and X. Zhang, "DOA estimation based on combined unitary ESPRIT for coprime MIMO radar," IEEE Communications Letters, vol. 21, no. 1, pp. 96-99, 2017.

[14] H. Jiang, J.-K. Zhang, and K. M. Wong, "Joint DOD and DOA estimation for bistatic MIMO radar in unknown correlated noise," IEEE Transactions on Vehicular Technology, vol. 64, no. 11, pp. 5113-5125, 2015.

[15] A. Khabbazibasmenj, A. Hassanien, S. A. Vorobyov, and M. W. Morency, "Efficient transmit beamspace design for search-free based DOA estimation in MIMO radar," IEEE Transactions on Signal Processing, vol. 62, no. 6, pp. 1490-1500, 2014.

[16] A. Hassanien and S. A. Vorobyov, "Transmit energy focusing for DOA estimation in MIMO radar with colocated antennas," IEEE Transactions on Signal Processing, vol. 59, no. 6, pp. 26692682, 2011

[17] P. Chen, P. Zhan, and L. Wu, "Clutter estimation based on compressed sensing in bistatic MIMO radar," IET Radar, Sonar \& Navigation, vol. 11, no. 2, pp. 285-293, 2017.

[18] M. L. Bencheikh and Y. Wang, "Joint DOD-DOA estimation using combined ESPRIT-MUSIC approach in MIMO radar," IEEE Electronics Letters, vol. 46, no. 15, pp. 1081-1083, 2010.

[19] A. Hassanien, S. A. Vorobyov, and A. Khabbazibasmenj, "Transmit radiation pattern invariance in MIMO radar with application to DOA estimation," IEEE Signal Processing Letters, vol. 22, no. 10, pp. 1609-1613, 2015.

[20] X. Zhang, L. Xu, and D. Xu, "Direction of departure (DOD) and direction of arrival (DOA) estimation in MIMO radar with reduced-dimension MUSIC," IEEE Communications Letters, vol. 14, no. 12, pp. 1161-1163, 2010.

[21] M. Cattenoz and S. Marcos, "Adaptive processing for MIMO radar realistic non perfectly orthogonal waveforms," in Proceedings of the 2014 IEEE Radar Conference, RadarCon 2014, pp. 1323-1328, Cincinnati, Ohio, USA, May 2014.

[22] G. Zheng, "DOA estimation in MIMO radar with non-perfectly orthogonal waveforms," IEEE Communications Letters, vol. 21, no. 2, pp. 414-417, 2017.

[23] P. Chen, C. Qi, L. Wu, and X. Wang, "Estimation of extended targets based on compressed sensing in cognitive radar system," IEEE Transactions on Vehicular Technology, vol. 66, no. 2, pp. 941-951, 2017.

[24] J.-F. Determe, J. Louveaux, L. Jacques, and F. Horlin, "On the noise robustness of simultaneous orthogonal matching pursuit," IEEE Transactions on Signal Processing, vol. 65, no. 4, pp. 864875,2017 


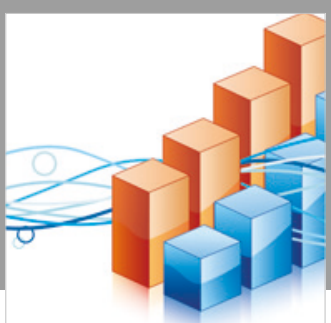

Advances in

Operations Research

\section{-n-m}
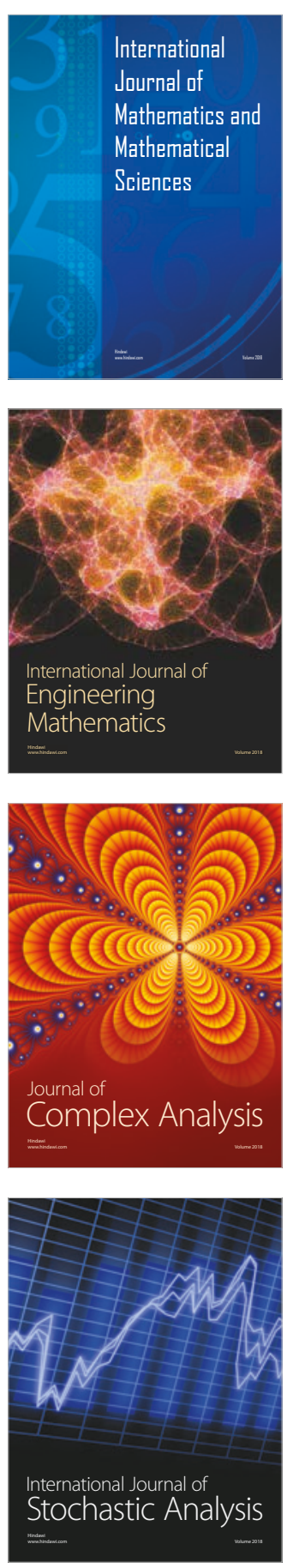
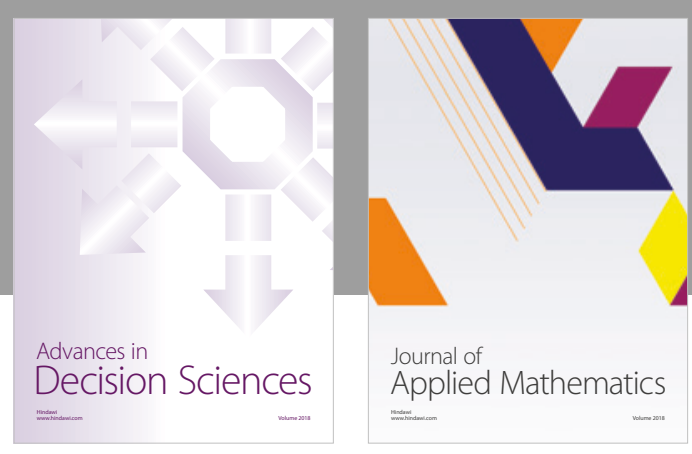

Journal of

Applied Mathematics
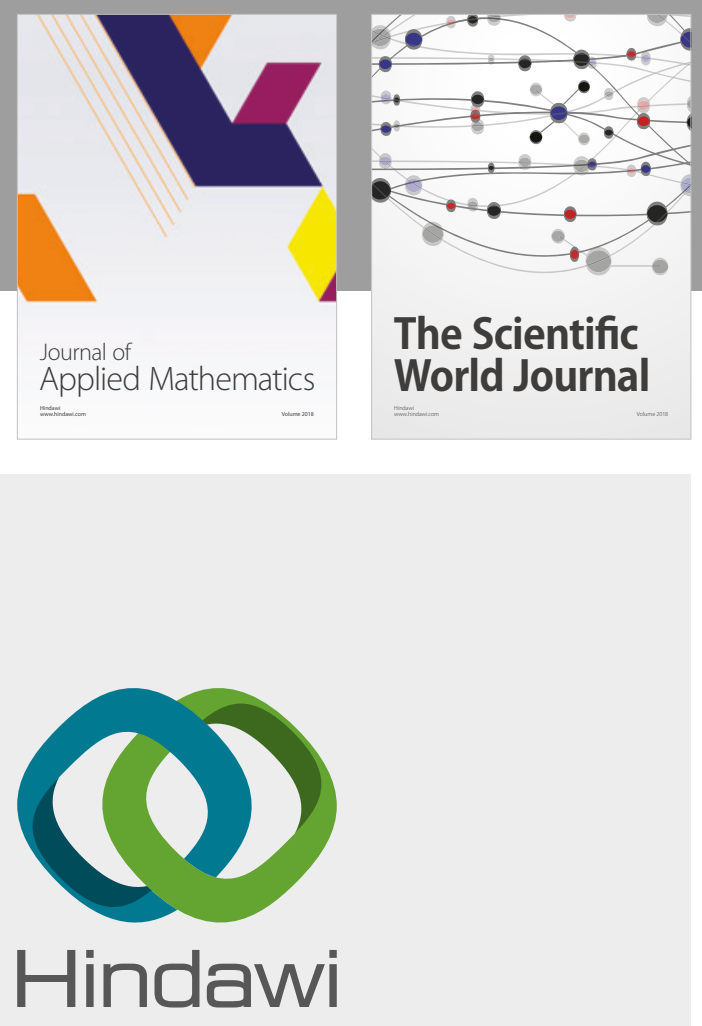

Submit your manuscripts at

www.hindawi.com

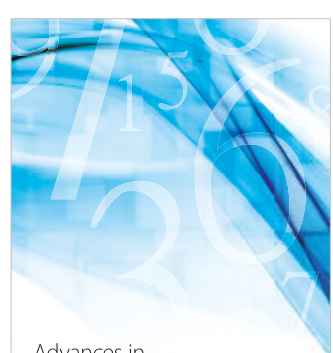

Advances in
Numerical Analysis
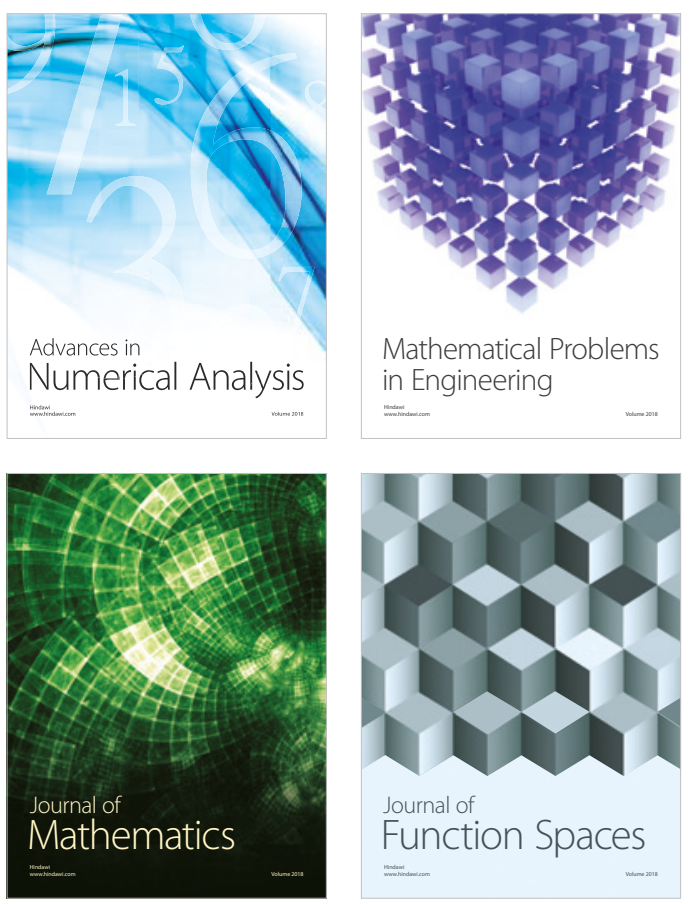

Mathematical Problems in Engineering

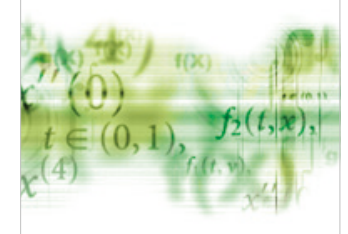

International Journal of

Differential Equations

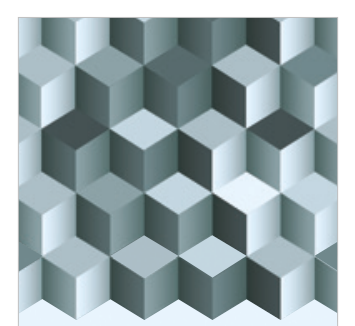

Journal of

Function Spaces

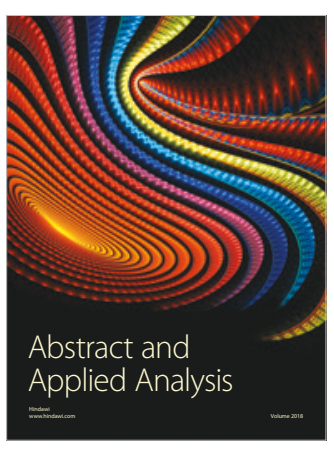

The Scientific

World Journal

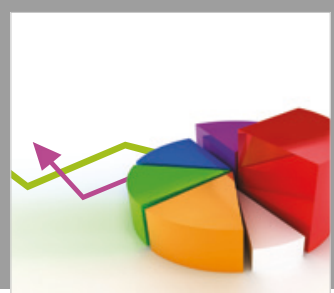

Journal of

Probability and Statistics
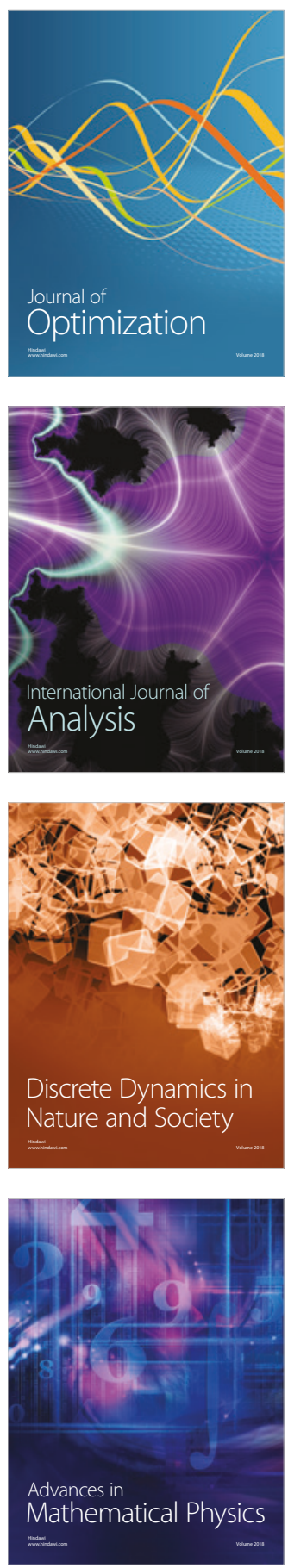